\title{
The explosion in cryptocurrencies: a black hole analogy
}

\author{
Antonis Ballis* ${ }^{*}$ and Konstantinos Drakos
}

\author{
${ }^{*}$ Correspondence: \\ aballis@aueb.gr \\ Department of Accounting \\ and Finance, School \\ of Business, Athens University \\ of Economics and Business, \\ 76 Patission Street, \\ 10434 Athens, Greece
}

\begin{abstract}
Using an analogy between finance and astrophysics, this study aims to investigate whether there exists a mechanism that can describe the explosive increase in the number of traded cryptocurrencies and the cryptocurrency market in general. In physics, the Schwarzschild radius indicates that black holes are constantly expanding because of their mass increase. Enriching this analogy, we consider the cryptocurrency market as a self-gravitational body whose mass is denoted by (1) the number of traded cryptocurrencies and (2) in terms of increasing market capitalization for a given number of traded cryptocurrencies. By analyzing weekly snapshot data of all traded cryptocurrencies from January 4, 2009, to June 14, 2020, we find evidence that the above-mentioned mechanism exists. The results clearly indicate the self-gravitational property of the cryptocurrency market, which is direct evidence toward the hypothesis that the changes in the traded cryptocurrencies are a positive function of the previous period's number of traded cryptocurrencies.
\end{abstract}

Keywords: Bitcoin, Cryptocurrencies, Econophysics, Negative specific heat, Thermodynamic disequilibrium

JEL Classification: G14, G15, G23

\section{Introduction}

The financial market universe comprises a wide set of assets that offer potential investors almost countless investment opportunities. New financial assets and instruments are formulated or developed periodically, which ultimately attracts capital and attention. The latest example is the cryptocurrency market, which started in 2009 with the then exotic, relative, and mysterious-but now well-known-Bitcoin (Nakamoto 2008). The emergence of the cryptocurrency market has merited a rapidly growing and robust academic literature, as shown by the systematic analysis of Xu et al. (2019). However, this study does not focus on the standard research issues relevant to the cryptocurrency market because they tend to overlook other equally important phenomena. Corbet et al. (2019) outlined that both quantitative and non-quantitative research on the cryptocurrency market is available, with the latter mostly focusing on regulation (Böhme et al. 2015; Hendrickson and Luther 2017), cybercriminality (Chao et al. 2019; Li et al. 2020; Vandezande 2017), and efficiency (Roth 2015). Regarding the quantitative analyses, as shown by Corbet et al. (2019), many extant studies on cryptocurrencies have source, provide a link to the Creative Commons licence, and indicate if changes were made. The images or other third party material in this article are included in the article's Creative Commons licence, unless indicated otherwise in a credit line to the material. If material is not included in the article's Creative Commons licence and your intended use is not permitted by statutory regulation or exceeds the permitted use, you will need to obtain permission directly from the copyright holder. To view a copy of this licence, visit http://creativecommons. org/licenses/by/4.0/. 
concentrated on market efficiency (Urquhart 2016; Vidal-Tomás and Ibañez 2018; Wei 2018; Neves 2020) and asset pricing bubbles (Cheah and Fry 2015; Corbet et al. 2018). Furthermore, other research areas, such as unusually high returns and the high volatility of the cryptocurrency market (Feng et al. 2018; Katsiampa 2017; Jalali and Heidari 2020), along with the herding behavior (Bouri et al. 2019; Vidal-Tomás et al. 2019; Ballis and Drakos 2020) and contagion (Antonakakis et al. 2019) related to it, are generally wellknown in both academia and practice.

However, nonprofessionals are most likely not aware of the exponential expansion of the number of traded cryptocurrencies. To gauge this expansion, the following can be considered. At the birth of the cryptocurrency market, the number of cryptocurrencies was equal to one and remained at that level until April 17, 2011. Subsequently, the number of traded cryptocurrencies started to soar, reaching the value of 50 on August 4, 2013, while it increased to 500 by October 26, 2014, and further increased to 1500 by February 25, 2018; it currently (as of June 14, 2020) stands at 2,670 traded cryptocurrencies (source: CoinMarketCap). In other words, since its birth, the cryptocurrency market has exhibited a $266,900 \%$ increase in terms of the number of traded currencies. It is difficult to think of another economic and/or financial phenomenon with an equivalent increase rate over a decade, possibly with the exceptions of the Tulip Mania (1619-1622) and the Mississippi Bubble (1716-1719).

Therefore, our focus is on the mechanism that can describe the observed behavior of the explosive increase in the number of traded cryptocurrencies using an analogy between finance and astrophysics, where the new cryptocurrency market is considered to represent a celestial body belonging to the universe of financial assets. According to Newton's universal gravitation law, in physical systems, a body exerts a gravitational force to its surroundings that is proportional to its mass (i.e., bodies with greater mass exhibit higher gravity). A certain class of bodies, known as self-gravitational bodies, has the natural tendency to be in thermodynamic disequilibrium owing to their negative specific heat status (Thirring 1970; Zia et al. 2002); thus, their gravitational force increases, leading to a spiral where increases in the mass further reinforce the gravitational force.

For instance, a similar phenomenon is observed for black holes. When a star dies, it collapses inward rapidly. The fact that the radiation in its core ceases to exist leads to gravity pushing in extreme volumes. Consequently, the star explodes into a supernova (Woosley and Weaver 1986), which is a catastrophic expulsion of its outer material (Lohnes 2018), leading the dying star into a continuum of collapse until it becomes a singularity (zero volume, infinite density). These singularities are the dead centers of black holes leading them to be "objects" with extreme gravity volumes that can even bend spacetime (Hawking 1975). At the edge of a black hole, or the event horizon (Hawking 1973), as it is called, an astronomical time deceleration is present. Karl Schwarzschild (1916) identified the limit at which gravity triumphs over the other physical forces, creating a black hole. Today, we call this number the Schwarzschild radius, which is expressed as

$$
R_{s}=\frac{2 G M}{c^{2}},
$$


where $G$ is the gravitational constant, $M$ is the mass of the black hole, and $c$ is the speed of light. The Schwarzschild radius indicates that black holes are constantly expanding because of the mass increase.

For celestial bodies, the definition of mass is clear. However, from a financial/economics perspective, defining the appropriate measure of mass is not a straightforward exercise. Therefore, we consider the cryptocurrency market as a self-gravitational body. Our initial conjecture was considering the notion of mass based on the number of traded assets, which corresponds to the extensive margin in economics terminology and measures horizontal market expansion. However, it is plausible that the gravitational force may not solely lead to the creation of new cryptocurrencies, but instead attract more capital toward the already existing traded cryptocurrencies. ${ }^{1}$ To operationalize this notion, we consider a different potential proxy for mass in terms of increasing market capitalization for a given number of traded cryptocurrencies. The rationale is that, apart from a horizontal expansion, a vertical expansion might also exist. To capture this vertical expansion, we resort to the intensive margin at any given point in time, defined as the total market capitalization divided by the total number of traded assets.

If the cryptocurrency market exhibits increasing entropy due to its negative specific heat, there exists a testable implication in its behavior. In particular, the mass of the cryptocurrency market, as captured by the number of traded cryptocurrencies (according to our first hypothesis regarding the definition of mass) at a point in time, determines its gravitational force. Then, the testable hypothesis is that, as the mass of the market (the number of traded cryptocurrencies) increases, the gravitational force increases, and, consequently, more cryptocurrencies are born (traded) in the next period. Accordingly, the same notion and testable hypothesis apply to the second definition of mass, denoted by the intensive margin.

Despite the rapidly growing academic literature on cryptocurrencies, to the best of our knowledge, such a phenomenon has not been formally investigated. This is exactly the gap of the academic literature that our analysis aims to fill, by investigating and measuring whether there exists a mechanism that can describe the explosive increase in the number of traded cryptocurrencies. The remainder of the paper is structured as follows. "Data and empirical methodology" section describes the dataset and provides a review of the models deployed in the analysis. "Empirical results" section presents and discusses the empirical findings. Finally, "Conclusions" section concludes.

\section{Data and empirical methodology}

The data are weekly snapshots of all traded cryptocurrencies from January 4, 2009, to June 14, 2020 (source: CoinMarketCap), for a total of 598 observations. Since April 17, 2011, when the second cryptocurrency made its appearance, the number of traded cryptocurrencies started to increase, as depicted in Fig. 1.

$M_{t}$ denotes the number of cryptocurrencies traded at time $t$, where $t$ spans the period from 2009 to 2020 at weekly intervals, and $\Delta M_{t}$ represents the change in the number of cryptocurrencies.

\footnotetext{
${ }^{1}$ We would like to thank one of the anonymous referees for pointing out this issue.
} 


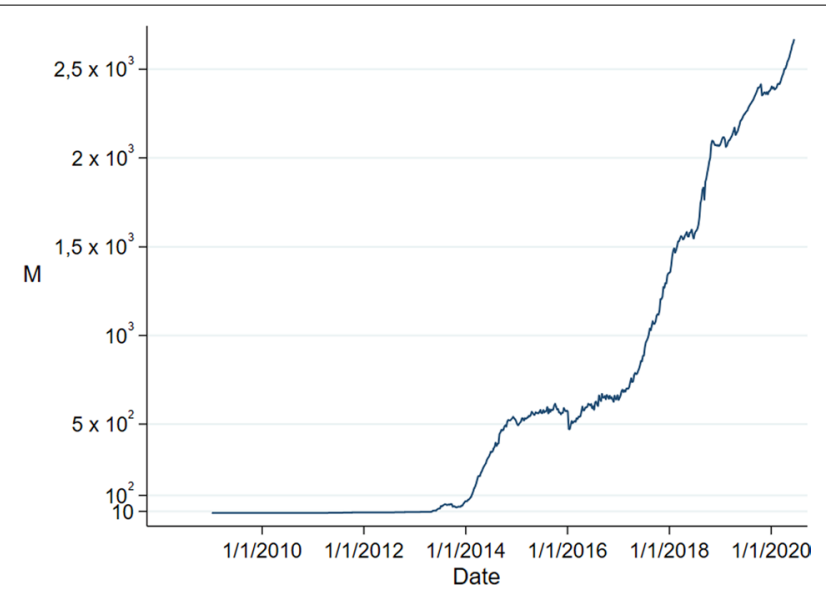

Fig. 1 Illustration of the number of Cryptocurrencies by date. Source: Own estimations

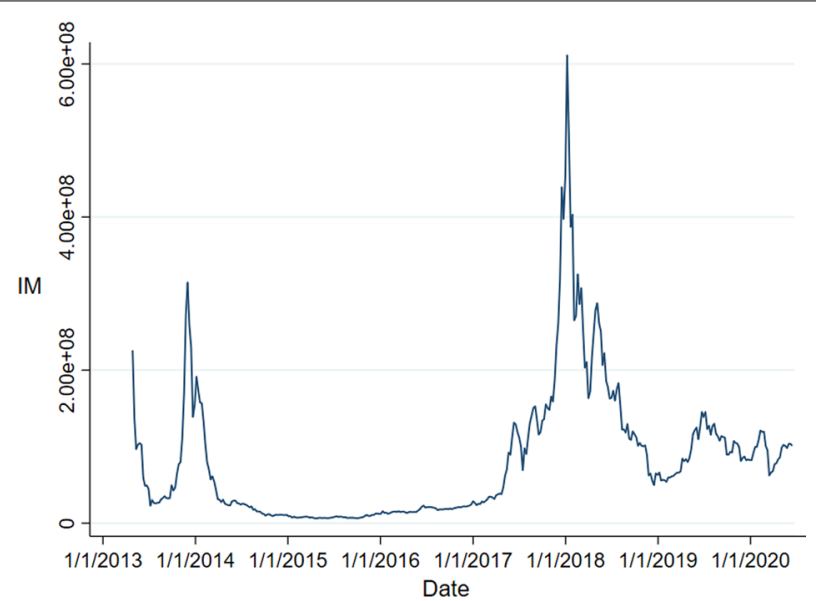

Fig. 2 Illustration of the market capitalization divided by the number of cryptocurrencies traded. Source: Own estimations

Figure 2 depicts the intensive margin, as market capitalization divided by the number of traded cryptocurrencies.

$I M_{t}$ denotes the market capitalization divided by the number of cryptocurrencies traded at time $t$, where $t$ spans the period from 2013 to 2020 at weekly intervals, and $\Delta\left(I M_{t}\right)$ is the change.

In Figs. 3 and 4, the lowess smoother graphs show a positive relationship between the above-mentioned parameters.

If the cryptocurrency market possesses a self-gravitational property, we expect that its gravitational force increases with mass. This behavior translates in a testable hypothesis, where the change $\left(\Delta M_{t}\right)$ in the number of traded cryptocurrencies should be a positive function of the previous period's number of traded cryptocurrencies $\left(M_{t-1}\right)$ :

$$
\Delta M_{t}=a+\beta * M_{t-1}+u_{t},
$$




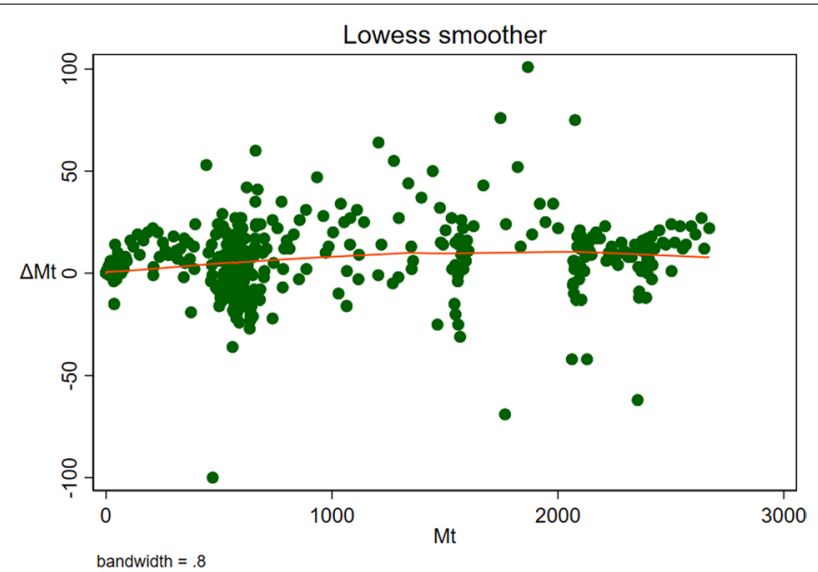

Fig. 3 Lowess smoother graph regressing the number of cryptocurrencies $\left(M_{t}\right)$ on the change of the number of cryptocurrencies $\left(\Delta M_{t}\right)$. Source: Own estimations

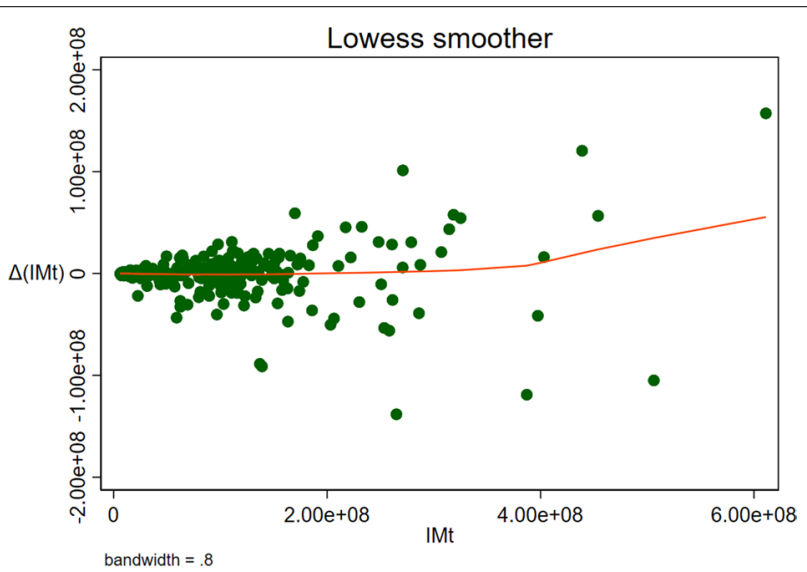

Fig. 4 Lowess smoother graph regressing market capitalization divided by the number of cryptocurrencies traded $I M_{t}$ on its change $\Delta\left(I M_{t}\right)$. Source: Own estimations

where $a$ and $\beta$ are the estimable parameters and $u_{t}$ is a well-behaved disturbance term. Furthermore, our prior compatible with the self-gravitational property is that $\beta>0$. Therefore, the testable hypothesis is formulated as

$$
\begin{aligned}
& H_{0}: \beta=0, \\
& H_{1}: \beta>0 .
\end{aligned}
$$

Accordingly, the second testable hypothesis is that the change $\Delta\left(I M_{t}\right)$ in market capitalization divided by the number of traded cryptocurrencies should be a positive function of the previous period $\left(I M_{t-1}\right)$ :

$$
\Delta\left(I M_{t}\right)=\gamma+\delta * I M_{t-1}+u_{t},
$$

where $\gamma$ and $\delta$ are the estimable parameters and $u_{t}$ is well-behaved disturbance term. In this case, the testable hypothesis is formulated as 
Table 1 Estimation results of $\Delta M_{t}=a+\beta * M_{t-1}+u_{t}$

\begin{tabular}{lll}
\hline & $\boldsymbol{a}$ & $\boldsymbol{\beta}$ \\
\hline Coef & $1.5751^{*}$ & $0.0044^{* * *}$ \\
& $(0.7609)$ & $(0.0007)$ \\
t-statistic & 2.07 & 6.06 \\
$p$ value & $(0.039)$ & $(0.000)$ \\
Obs & R-squared & Adj R-squared \\
597 & 0.0581 & 0.0565 \\
\hline
\end{tabular}

$\left(*^{* *}\right),\left({ }^{* *}\right),\left({ }^{*}\right)$ denotes that the coefficient is significant at the $1 \%, 5 \%, 10 \%$ level

Table 2 Estimation results of $\Delta\left(I M_{t}\right)=\gamma+\delta * I M_{t-1}+u_{t}$

\begin{tabular}{lll}
\hline & $\boldsymbol{\gamma}$ & $\boldsymbol{\delta}$ \\
\hline Coef & $2,531,778$ & $-0.0356^{* *}$ \\
& $(1,545,242)$ & $(0.013)$ \\
t-statistic & 1.64 & -2.74 \\
$p$ value & $(0.102)$ & $(0.006)$ \\
Obs & R-squared & Adj R-squared \\
372 & 0.0199 & 0.0172 \\
\hline
\end{tabular}

$(* * *),(* *),(*)$ denotes that the coefficient is significant at the $1 \%, 5 \%, 10 \%$ level

$$
\begin{aligned}
& H_{0}: \delta=0 \\
& H_{1}: \delta>0
\end{aligned}
$$

Here, $\beta=0$ implies that the growth of traded cryptocurrencies does not depend on the past number of traded cryptocurrencies. If $\beta>0$, the growth increases as the number of previous period's cryptocurrencies is higher. The same applies for the second notion regarding mass and $\delta=0$ and $\delta>0$.

The estimation of and inference for the above unknown parameters is a straightforward exercise provided that the variables are stationary. However, from the previous section, $M_{t}$ and $I M_{t}$ are clearly non-stationary; therefore, the standard asymptotic theory does not apply. Coincidently, the models above [Eqs. (1) and (2)] are identical to the standard Dickey-Fuller formulation (Dickey and Fuller 1979).

\section{Empirical results}

Equations (1) and (2) were used to assess whether the cryptocurrency market possesses a self-gravitational property. If so, as previously stated, it is expected that the market's gravitational force will tend to increase with its mass. Tables 1 and 2 report the results for our models using ordinary least squares estimations.

In the first specification, where $\Delta M_{t}$ is the dependent variable, we find that the main parameter of interest $(\beta)$ is positive and statistically significant at the $1 \%$ level.

In the second specification, where $\Delta\left(I M_{t}\right)$ is the dependent variable, we find that the main parameter of interest $(\delta)$ is negative and statistically significant at the $5 \%$ level.

Based on Eq. (1), which allows to draw conclusion about the stationarity of $M_{t}$, our findings show that the past level of $M_{t}$ exerts a positive impact on the change in the number of cryptocurrencies $\left(\Delta M_{t}\right)$. By contrast, the results of Eq. (2) suggest that the past level of $I M_{t}$ does not only exert a positive impact but also exerts a negative 
impact on $\Delta\left(I M_{t}\right)$ based on the point estimates. Therefore, based on our empirical findings, even if the notion examined above is plausible, it is not in operation. Furthermore, the negative sign indicates that the intensive margin does not only act as a gravitational force in the sense that more capital goes toward existing cryptocurrency assets. However, even if more capital is attracted, it is diffused over a growing number of assets. Insofar, the number of traded currencies is a manifestation of mass, meaning our results are compatible with the notion that the cryptocurrency market possesses a self-gravitational property.

\section{Conclusions}

This study aimed to assess for the first time, to the best of our knowledge, whether a mechanism exists that can describe the observed behavior of the explosive increase in the number of traded cryptocurrencies. The testable hypothesis was that, as the mass of the market [number of traded cryptocurrencies in Eq. (1) and market capitalization in Eq. (2)] increases, more cryptocurrencies are created (traded) in the next period [Eq. (1)], and more money are attracted onto the market [Eq. (2)]. The analysis was based on weekly data of the number of existing cryptocurrencies, along with their market capitalization.

From our results, we can infer that such a mechanism exists. This study identified a statistically significant positive $\beta(0.0044)$ coefficient, indicating a self-gravitational property, which is direct evidence toward the hypothesis that the change $\left(\Delta M_{t}\right)$ in the number of traded cryptocurrencies is, in fact, a positive function of the previous period's number of traded cryptocurrencies $\left(M_{t-1}\right)$. Additionally, we found a statistically significant negative $\delta(-0.0356)$; this indicates that not only does the intensive margin act as a gravitational force of the additional capital toward existing cryptocurrency assets but also is a clear indicator that new capital is diffused over a growing number of assets. One possible explanation is that market capitalization and, subsequently, $I M_{t}$ are only demand driven, whereas the number of cryptocurrencies-denoted as $M_{t}$-considers both demand and supply. In summary, these results are in agreement with our initial analogy that the cryptocurrency market is in a state of thermodynamic disequilibrium.

A fruitful and promising expansion of these results relates to improving the measurement of thermodynamic disequilibrium by the direct usage of entropy measures.

\section{Acknowledgements}

Not applicable.

Authors' contributions

$\mathrm{AB}$ : conceptualization, formal analysis, writing, review and editing, data curation, software. KD: conceptualization, formal analysis, writing and editing, project administration, supervision. Both authors read and approved the final manuscript.

\section{Funding}

This research is co-financed by Greece and the European Union (European Social Fund- ESF) through the Operational Programme «Human Resources Development, Education and Lifelong Learning» in the context of the project "Strengthening Human Resources Research Potential via Doctorate Research" (MIS-5000432), implemented by the State Scholarships Foundation (IKY).

\section{Availability of data and materials}

The selected database to be considered by the model was built with data found in CoinMarketCap (https://www.coinm arketcap.com). The data on which this study is based refer to weekly snapshots of all traded cryptocurrencies from January 4, 2009 to June 14, 2019. 


\section{Competing interests}

The authors declare that they have no competing interests.

Received: 7 May 2020 Accepted: 11 December 2020

Published online: 27 January 2021

\section{References}

Antonakakis N, Chatziantoniou I, Gabauer D (2019) Cryptocurrency market contagion: market uncertainty, market complexity, and dynamic porfolios. J Int Financ Mark Inst Money 61:37-51. https://doi.org/10.1016/j.intfin.2019.02.003

Ballis A, Drakos K (2020) Testing for herding in the cryptocurrency market. Finance Res Lett 33:101210. https://doi. org/10.1016/j.frl.2019.06.008

Böhme R, Christin N, Edelman B, Moore T (2015) Bitcoin: economics, technology, and governance. J Econ Perspect 29(2):213-238. https://doi.org/10.1257/jep.29.2.213

Bouri E, Gupta R, Roubaud D (2019) Herding behaviour in cryptocurrencies. Finance Res Lett 29:216-221. https://doi. org/10.1016/j.frl.2018.07.008

Chao X, Kou G, Peng Y, Alsaadi FE (2019) Behavior monitoring methods for trade-based money laundering integrating macro and micro prudential regulation: a case from China. Technol Econ Dev Econ 25(6):1081-1096. https://doi. org/10.3846/tede.2019.9383

Cheah E-T, Fry J (2015) Speculative bubbles in bitcoin markets? An empirical investigation into the fundamental value of bitcoin. Econ Lett 130:32-36. https://doi.org/10.1016/j.econlet.2015.02.029

Corbet S, Lucey B, Yarovaya L (2018) Datestamping the bitcoin and ethereum bubbles. Finance Res Lett 26:81-88. https:// doi.org/10.1016/j.frl.2017.12.006

Corbet S, Lucey BM, Urquhart A, Yarovaya L (2019) Cryptocurrencies as a financial asset: a systematic analysis. Int Rev Financ Anal 62:182-199. https://doi.org/10.1016/j.irfa.2018.09.003

Dickey DA, Fuller WA (1979) Distribution of the estimators for autoregressive time series with a unit root. J Am Stat Assoc 74:427-431. https://doi.org/10.2307/2286348

Feng W, Wang Y, Zhang Z (2018) Informed trading in the bitcoin market. Finance Res Lett 26:63-70. https://doi. org/10.1016/j.frl.2017.11.009

Hawking SW (1973) Event horizon. Gordon and Breach, Science Publishers Inc., New York

Hawking SW (1975) Particle creation by black holes. Commun Math Phys 43:199-220. https://doi.org/10.1007/BF023 45020

Hendrickson JR, Luther WJ (2017) Banning bitcoin. J Econ Behav Organ 141:188-195. https://doi.org/10.1016/j jebo.2017.07.001

Jalali MHM, Heidari H (2020) Predicting changes in bitcoin price using grey system theory. Financ Innov 6:13. https://doi. org/10.1186/s40854-020-0174-9

Katsiampa P (2017) Volatility estimation for bitcoin: a comparison of garch models. Econ Lett 158:3-6. https://doi. org/10.1016/j.econlet.2017.06.023

Li X, Jiang P, Chen T, Luo X, Wen Q (2020) A survey on the security of blockchain systems. Future Gener Comput Syst 107:841-853. https://doi.org/10.1016/j.future.2017.08.020

Lohnes K (2018) How do black holes really work? https://www.britannica.com/story/how-do-black-holes-really-work Nakamoto S (2008) Bitcoin: a peer-to-peer electronic cash system. Cryptography Mailing list at https://metzdowd.com

Neves RH (2020) Bitcoin pricing: impact of attractiveness variables. Financ Innov 6:21. https://doi.org/10.1186/s4085 4-020-00176-3

Roth N (2015) An architectural assessment of bitcoin: using the systems modeling language. Procedia Comput Sci 44:527-536. https://doi.org/10.1016/j.procs.2015.03.066

Schwarzschild K (1916) On the gravitational field of a mass point according to Einstein's theory. Sitzungsber.Preuss.Akad. Wiss.Berlin (Math.Phys.), 189-196

Thirring W (1970) Systems with negative specific heat. Z Physik 235:339-352. https://doi.org/10.1007/BF01403177

Urquhart A (2016) The inefficiency of bitcoin. Econ Lett 148:80-82. https://doi.org/10.1016/j.econlet.2016.09.019

Vandezande N (2017) Virtual currencies under EU anti-money laundering law. Comput Law Secur Rev 33(3):341-353. https://doi.org/10.1016/j.clsr.2017.03.011

Vidal-Tomás D, Ibañez A (2018) Semi-strong efficiency of bitcoin. Finance Res Lett 27:259-265. https://doi.org/10.1016/j. frl.2018.03.013

Vidal-Tomás D, Ibáñez AM, Farinós JE (2019) Herding in the cryptocurrency market: CSSD and CSAD approaches. Finance Res Lett 30:181-186. https://doi.org/10.1016/j.frl.2018.09.008

Wei WC (2018) Liquidity and market efficiency in cryptocurrencies. Econ Lett 168:21-24. https://doi.org/10.1016/j.econ et.2018.04.003

Woosley SE, Weaver TA (1986) The physics of supernova explosions. Ann Rev Astron Astrophys 24:205-253. https://doi. org/10.1146/annurev.aa.24.090186.001225

Xu M, Chen X, Kou G (2019) A systematic review of blockchain. Financ Innov 5:27. https://doi.org/10.1186/s4085 4-019-0147-z

Zia RKP, Præstgaard E, Mouritsen OG (2002) Getting more from pushing less: negative specific heat and conductivity in non-equilibrium steady states. Am J Phys 70:384-392

\section{Publisher's Note}

Springer Nature remains neutral with regard to jurisdictional claims in published maps and institutional affiliations. 